\title{
Tribo-electrification of pharmaceutical powder blends
}

\author{
Antonella Rescaglio ${ }^{a, b}$, Frederic De Smet ${ }^{c}$, Luc Aerts ${ }^{c}$, and Geoffoy Lumay ${ }^{a, b}$ \\ ${ }^{a}$ CESAM - APTIS, University of Liege, Liège, Belgium; ${ }^{b}$ CESAM - GRASP, University of Liege, Liège, Belgium; ${ }^{C}$ UCB Pharma, Braine- \\ I'Alleud, Belgium
}

\begin{abstract}
It is well known in industrial applications involving powders and granular materials that the presence of electrostatic charges influences drastically the material flowing properties. The triboelectric charges are produced during flow at the contacts between the grains and at the contacts between the grains and the container. Unfortunately, the triboelectric effect is still poorly understood, even at the fundamental level. Therefore, the approach to solve practical problems is mostly empirical. Moreover, reproducible electrostatic measurements are difficult to perform. In the present study, the ability of a set of excipients and active pharmaceutical ingredients (APIs) to produce electrostatic charges during flow in contact with different materials is analyzed with a recently developed instrument called GranuCharge. While different excipients have almost the same triboelectric behavior and a low chargability, APls show complex triboelectric properties. Some APIs charge a lot while other APIs charge less. Afterward, the electrostatic behavior of API/ excipient blends is considered. We show that the net charge of the blend is a complex function of the relative quantity of API in the mixture. Moreover, both the quantity and the sign of the charge are found to depend on the material in contact with the powder during the flow.
\end{abstract}

\section{KEYWORDS}

Electrostatic charging; powder; particle/particulate; mixing; electrical properties; tester/testing; measurement

\section{Introduction}

Roughly $80 \%$ of the pharmaceutical products in US are tablets and capsules involving powders and granular materials, Muzzio, Shinbrot, and Glasser (2002). In addition, more recent and more complex formulations like dry powder inhalers (DPI) are based on complex powder technology. Moreover, in order to avoid problems, it is important for pharmaceutical companies to improve the economical efficacy of their production processes. A robust manufacturing process involving powder requires reliable powder flow properties. Unfortunately, pharmaceutical powders are usually cohesive and a deep understanding of their properties needs a good knowledge of the cohesive forces acting between the grains, Sarkar, Mukherjee, and Chaudhuri (2017). This knowledge is strongly multidisciplinary and there are still many open questions remaining, for example, relating to electrostatic powder properties.

When two materials are rubbed, electric charges are exchanged at the surfaces, Lacks (2010); Matsusaka et al. (2010). This contact electrification is an old fundamental scientific subject. However, despite the numerous studies dedicated to this subject, the fundamental mechanisms behind the triboelectric effect are not fully understood. In particular, the charging of objects composed by the same material, Waitukaitis et al. (2014), and charging of powders, Matsusaka et al. (2010); Mersch et al. (2010); Ireland and Nicholson (2011) are subjects needing deeper investigations. Even the basic question related to the nature of the transferred charges (electrons, ions, or material) is still debated, Mehrani, Murtomaa, and Lacks (2017). The difficulties are related to the non-equilibrium character of the triboelectric exchange and to the variety of mechanisms behind this effect. Indeed, different mechanisms could induce triboelectric charges inside powders: impact, rolling, and sliding (or rubbing), Ireland (2010). Moreover, the nature of contact inside a powder (normal forces, contact areas, ...) is difficult to quantify.

Pharmaceutical powders can accumulate electrostatic charges during manipulations in the industry and also during clinical use. The presence of electrostatic charges in powder induces adhesion, Ghori, Šupuk, and Conway (2015), and cohesion leading to intermittent or blocked flow and consequently non-uniformity of dosage. In DPI application, electrostatic forces may induce undesirable adhesion of drug particles to the device and can affect the trajectory of the aerosol in the lung, Wong, Chan, and Kwok (2013). As active pharmaceutical ingredients (APIs) and excipients are organic materials with high resistivity, charge decay takes minutes or hours and an accumulation of charge is induced by powder manipulations. In addition to flow problems, electrostatic discharge could ignite fires and explosions when small grains are dispersed in air, Glor (2003); Ohsawa (2011). Therefore, a better understanding of charging mechanisms could improve the development and production processes and also the delivery systems. The factors influencing the charging of a powder in contact with a surface 
are multiple: surface nature (material, roughness, and surface cleanness), grain properties (chemical nature, grain size distribution, grain shape and roughness, grain crystallinity), and environmental conditions (RH). For a review, see reference Wong, Kwok, and Chan (2015). Due to the complexity, our understanding of powder electrostatics is far from complete and further research is needed.

In some applications, tribo-electrification causes complications. However, the triboelectric effect can be used advantageously in other applications (electrophotography, powder coating processes, triboelectric separators, agglomeration), Lachiver et al. (2006). In pharmaceutical applications, charging APIs and excipients with opposite charges can help to reinforce blend homogeneity by inducing attraction between the constituents, Lachiver et al. (2006).

Different studies dedicated to triboelectric charges in granular materials have been performed with model systems (typically monodisperse, spherical, and non-cohesive grains), Ireland (2010); Mersch et al. (2010); Waitukaitis et al. (2014). These fundamental studies are necessary to improve the understanding of the tribocharging mechanisms. However, the powders used in industry are more complex due to size distribution, complex grain shape, cohesive forces, and water content, Lumay et al (2012); Boschini et al (2015). Moreover, the combination of different grain types is expected to lead to highly complex triboelectric behavior. Finally, the material in contact with the powder during processing can play a role too.

Engers et al. (2007) investigated tribocharging of pharmaceutical mixtures in a stainless steel blender. The charge of the mixture was found to be lower than the charge of the individual components. Murtomaa and Laine (2000) studied tribocharging of lactose/glucose mixtures in a glass pipe. They observed a charge reversal: while the pure components charge positively, some mixtures were found to charge negatively. Elajnaf, Carter, and Rowley (2006) investigated the triboelectrification of micronized drug and lactose mixtures during mixing in cylindrical vessels made of stainless steel, polypropylene, and acetal at variable relative humidity. The charge was found to depend on both the nature of the powder and the surface in contact. Moreover, a coating of the mixer with drug or excipient was found to modify the creation of charges. Very recently, Zafar, Alfano, and Ghadiri (2018) presented a new dispersion technique for assessing triboelectric charging of powders. Finally, Naik, Mukherjee, and Chaudhuri (2016) have made a short review of the literature dedicated to granular mixture tribocharging and conclude that despite the reasonable number of investigations, the current knowledge on the triboelectric charging of mixtures is quite limited. In addition, the experimental setups used to perform the studies are customized devices and the results are difficult to compare. Therefore, a normalized method and a normalized measurement protocol are needed. This method needs to be practically usable with both freeflowing and highly cohesive powders. Moreover, the surface in contact with the powder during the charging process must be easy to modify.
In the present article, we describe a recently developed experimental method to measure the quantity of electric charges created inside a powder during a bulk flow in contact with a selected material. The device, called GranuCharge, is used to measure the triboelectric behavior of excipient/API blends. The excipients and the APIs selected for the present study are effectively used in the pharmaceutical industry. After the analysis of the triboelectric behavior of both the excipient and the APIs alone, we present original results evidencing the complexity of the tribocharging mechanism in powder blends. The influence of the relative air humidity $(\mathrm{RH})$ is also evidenced.

\section{Materials and methods}

\subsection{Materials}

A set of excipients widely used in industry and a set of APIs has been considered. In Table 1, the powders are listed and sorted as a function of their chargability against stainless steel (see hereafter). Five families of excipients are considered: microcrystalline cellulose (Avicel $^{\circledR}$ PH101, Avicel ${ }^{\circledR}$ PH102, and Avicel ${ }^{\circledR}$ DG from FMC), lactose monohydrate (Pharmatose $^{\circledR} 100 \mathrm{M}$ and Pharmatose ${ }^{\circledR} 200 \mathrm{M}$ from DFE pharma, Tablettose ${ }^{\circledR} 80$ and InhaLac ${ }^{\circledR} 230$ from Meggle pharma), mannitol (Pearlitol ${ }^{\circledR} 300$ DC, 400 DC, and 200 SD from Roquette), starch and maltitol (GalenIQ ${ }^{\mathrm{TM}} 800$ and GalenIQ $^{\mathrm{TM}} 721$ from Beneo). The main parameters extracted from grain size distributions measured in dry mode with a laser diffraction particle size analyzer (Malvern, Mastersizer 2000, Scirocco dispenser) are summarized in Table 2. The measurements were performed based on the MIE theory with the use of the general purpose calculation model (normal sensitivity). The samples are analyzed in triplicate and the data presented correspond to the average value. The pressures are indicated in Table 2. The vibration of the feeder is set at $70 \%$ for all samples. A set of 9 APIs from UCB Pharma has been selected (Table 1 and Figure 1). The main chemical characteristics of these APIs are shown in Figure 1 and the clinical uses are listed below.

Table 1. List of excipients and APIs considered in the present study.

\begin{tabular}{|c|c|c|c|c|c|}
\hline \multicolumn{2}{|c|}{ Excipients } & \multicolumn{4}{|c|}{ APIs } \\
\hline 1 & Pearlitol ${ }^{\circledR} 200$ SD & - & 15 & Mesna & - \\
\hline 2 & InhaLac ${ }^{\circledR} 230$ & - & 16 & Piracetam & - \\
\hline 3 & Avicel ${ }^{\circledR} \mathrm{PH} 101$ & - & 17 & Levetiracetam & - \\
\hline 4 & Pharmatose $^{(B)} 200 \mathrm{M}$ & - & 18 & Brivaracetam & + \\
\hline 5 & Tablettose $^{\circledR} 80$ & - & 19 & Rotigotine & + \\
\hline 6 & GalenIQ $^{\mathrm{TM}} 721$ & - & 20 & Lacosamide & + \\
\hline 7 & Avicel ${ }^{\circledR}$ DG & - & 21 & Cetirizine & + \\
\hline 8 & Pharmatose $^{\circledR} 100 \mathrm{M}$ & - & 22 & Hydroxyzine & + \\
\hline 9 & Sweetpearl ${ }^{\circledR}$ P200 & - & 23 & Levocetirizine & + \\
\hline 10 & GalenIQ $^{\mathrm{TM}} 800$ & - & & & \\
\hline 11 & Avicel $^{(} \mathrm{PH} 102$ & - & & & \\
\hline 12 & Pearlitol $^{\circledR} 300 \mathrm{DC}$ & - & & & \\
\hline 13 & Pearlitol $^{\circledR} 400$ DC & - & & & \\
\hline 14 & Maize starch & - & & & \\
\hline
\end{tabular}

The powders are within their category sorted (from the smallest to the highest charge, taking the sign into account) as a function of their electrification against stainless steel. The charge sign against stainless steel is also indicated. 
- Rotigotine ((S)-6-[propyl(2-thiophen-2-ylethyl)amino]$5,6,7,8$ - tetrahydronaphthalen-1-ol; trade name Neupro ${ }^{\circledR}$ ) is a dopamine agonist of the non-ergoline class of medications indicated for the treatment of Parkinson's disease and restless legs syndrome, Bunten and Happe (2006).

- Levocetirizine and Cetirizine are Cetirizine dihydrochloride (racemic mixture of $( \pm)$-[2-[4-[(4-chlorophenyl)phenylmethyl]-1- piperazinyl]ethoxy]acetic acid), sold under the brand name Zyrtec ${ }^{\circledR}$ among others, is a second generation antihistamine used in the treatment of hay fever, allergies, angioedema, and urticaria, Baltes, De Lannoy, and Rodriguez (1985).

- Mesna (sodium 2-sulfanylethanesulfonate) sold under the brand name Mesnex ${ }^{\circledR}$ among others, is a medication

Table 2. Main parameters extracted from grain size distributions obtained with the laser diffraction method.

\begin{tabular}{|c|c|c|c|c|c|}
\hline & $\begin{array}{c}\mathrm{d}(\mathrm{v}, 0.1) \\
\mu \mathrm{m}\end{array}$ & $\begin{array}{c}\mathrm{d}(\mathrm{v}, 0.5) \\
\mu \mathrm{m}\end{array}$ & $\begin{array}{c}\mathrm{d}(\mathrm{v}, 0.9) \\
\mu \mathrm{m}\end{array}$ & $\begin{array}{c}\text { Pressure } \\
\text { bar }\end{array}$ & \\
\hline Pearlitol $^{(} 200$ SD & 98 & 164 & 267 & 0.1 & Excipients \\
\hline InhaLac ${ }^{\circledR} 230$ & 59 & 98 & 152 & 0.1 & \\
\hline Avicel $^{\circledR} \mathrm{PH} 101$ & 19 & 51 & 107 & 3 & \\
\hline Pharmatose $^{\circledR} 200 \mathrm{M}$ & 14 & 53 & 136 & 0.1 & \\
\hline Tablettose ${ }^{\circledR} 80$ & 56 & 191 & 434 & 0.1 & \\
\hline GalenIQ $^{\mathrm{TM}} 721$ & 68 & 180 & 378 & 0.1 & \\
\hline Avicel $^{\circledR}$ DG & 16 & 45 & 121 & 3 & \\
\hline Pharmatose $^{\circledR} 100 \mathrm{M}$ & 86 & 169 & 291 & 0.1 & \\
\hline Sweetpearl ${ }^{\circledR}$ P200 & 118 & 284 & 553 & 0.1 & \\
\hline GalenIQ $^{\mathrm{TM}} 800$ & 12 & 34 & 85 & 0.1 & \\
\hline Avicel $^{\circledR} \mathrm{PH} 102$ & 33 & 99 & 210 & 3 & \\
\hline Pearlitol ${ }^{\circledR} 300 \mathrm{DC}$ & 222 & 337 & 510 & 0.1 & \\
\hline Pearlitol ${ }^{\circledR} 400 \mathrm{DC}$ & 271 & 424 & 662 & 0.1 & \\
\hline Maize starch & 9 & 14 & 21 & 3 & \\
\hline Mesna & 5 & 27 & 161 & 2.0 & APIs \\
\hline Piracetam & 78 & 216 & 483 & 2.0 & \\
\hline Levetiracetam & 23 & 111 & 289 & 2.0 & \\
\hline Brivaracetam & 66 & 225 & 499 & 1.5 & \\
\hline Rotigotine & 39 & 254 & 494 & 2.0 & \\
\hline Lacosamide & 4 & 15 & 111 & 2.0 & \\
\hline Hydroxyzine & 1 & 3 & 25 & 4.0 & \\
\hline Cetirizine & 2 & 7 & 254 & 2.5 & \\
\hline Levocetirizine & 1 & 4 & 180 & 2.5 & \\
\hline
\end{tabular}

used to reduce the incidence of hemorrhagic cystitis and hematuria when a patient receives ifosfamide or cyclophosphamide for cancer chemotherapy, Shaw and Graham (1987). Mesna is also used as a mucolytic agent, working in the same way as acetylcysteine; it is sold for this indication as Mistabron ${ }^{\circledR}$, van de Walle, Lauwers, and Adriaensen (1976).

- Levetiracetam ((2S)-2-(2-oxopyrrolidin-1-yl)butanamide; trade name Keppra $\left.{ }^{\circledR}\right)$, is a widely used antiepileptic drug that provides protection against partial seizures and is also effective in the treatment of primarily generalized seizure syndromes including juvenile myoclonic epilepsy, Rogawski (2008).

- Brivaracetam ((2S)-2-[(4R)-2-oxo-4-propylpyrrolidin-1yl] butanamide; trade name Briviact $\left.{ }^{\circledR}\right)$, a chemical analog of levetiracetam, is also an antiepileptic drug used for uncontrolled partial epilepsy in adults, Matagne et al. (2008).

- Hydroxyzine dihydrochloride, (( \pm$)-2-(2-\{4-[(4-c h l o r o-$ phenyl)-phenylmethyl]piperazin-1-yl\}ethoxy)ethanol) sold under the brand name Atarax among others, is a firstgeneration antihistamine. It is also an effective antiemetic, sedative, hypnotic, and anxiolytic drug, Guaiana, Barbui, and Cipriani (2010).

- Piracetam (2-(2-Oxopyrrolidin-1-yl)acetamide; trade name Nootropil $\left.{ }^{\mathbb{B}}\right)$, is a nootropic drug used in the therapy of age-related cognitive disturbances and poststroke aphasia. Clinical experience has also shown that at high doses, it is effective against cortical myoclonus, Genton and van Vleymen (2000).

- Lacosamide((R)-2-(acetylamino)-N-benzyl-3-methoxypropanamide; trade name Vimpat $\left.{ }^{\circledR}\right)$ is an antiepileptic medication for the adjunctive treatment of partial-onset seizures and diabetic neuropathic pain, Hoy (2013).

Figure 2 presents a selection of SEM micrographs of the powders considered to make the blends (see hereafter) (SEM from JEOL, JSM-IT300LV)). The micrographs of the APIs

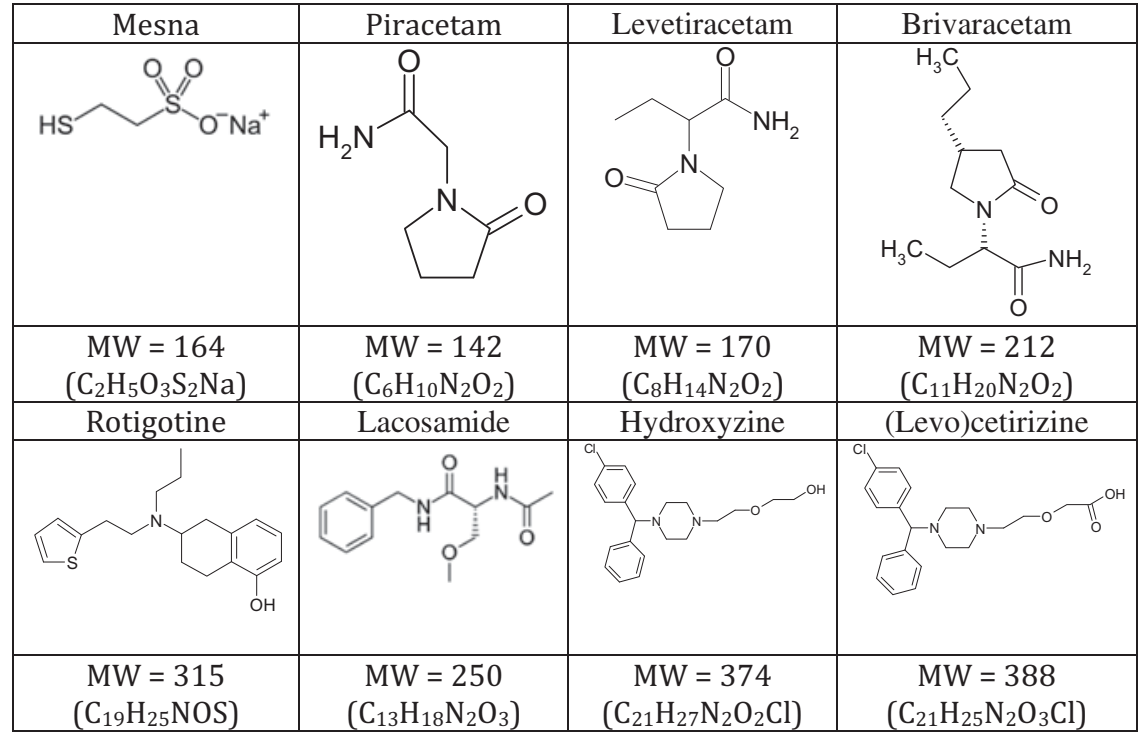

Figure 1. Molecular characteristics of the APIs. 

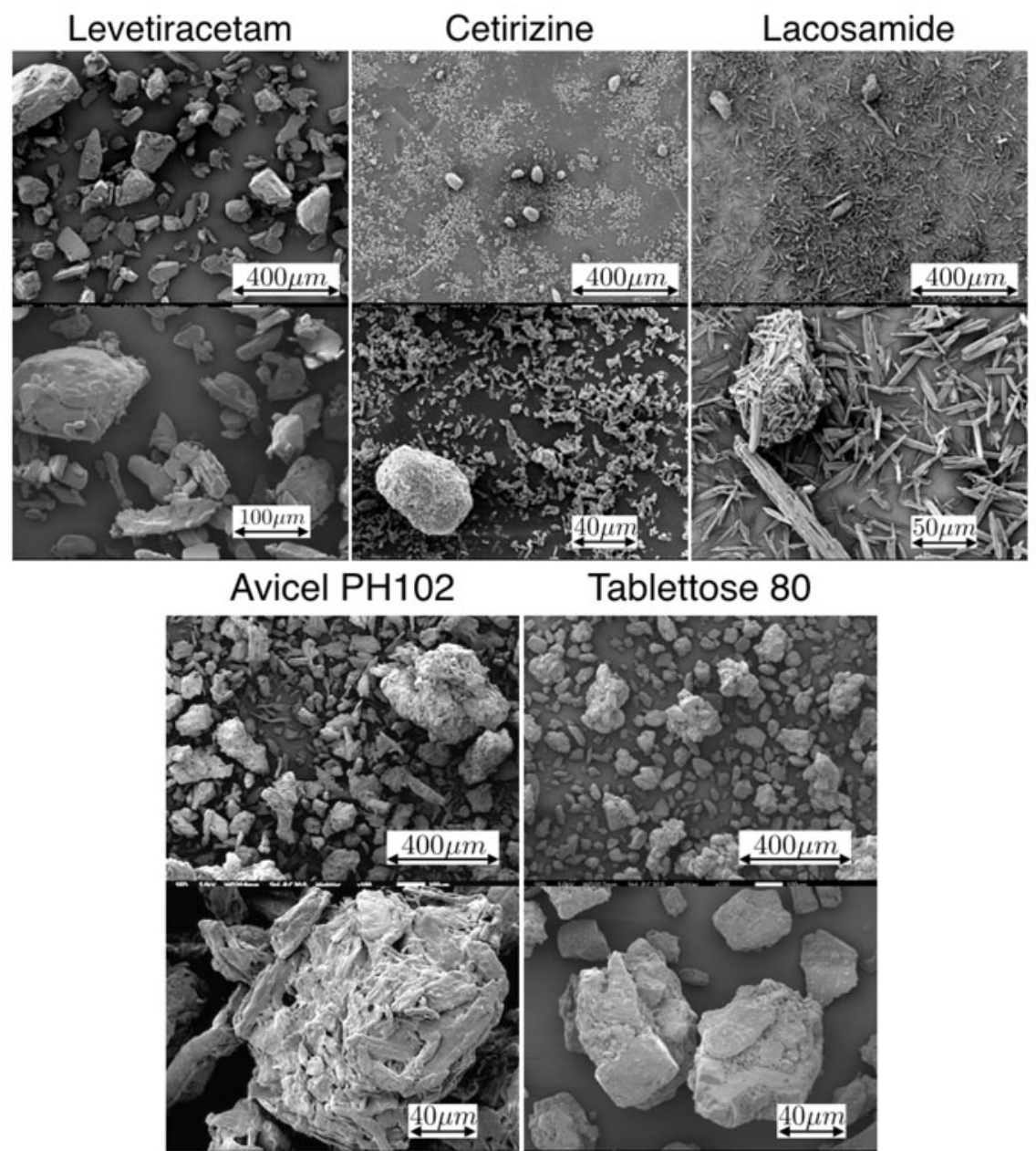

Figure 2. SEM micrographs of the powders considered to make the blends. (top) APIs (bottom) Excipients. Two magnifications are presented for each powder.

are showing that they have different grain sizes and shapes. Levetiracetam, Avicel, and Tablettose powders are made of quite large blocks, Cetirizine is made of very small particles with the presence of some agglomerates and lacosamide is made of needles also with some agglomerates.

\subsection{Methods}

A powder tribocharger (see Figure 3) is used to measure the total electrostatic charge created inside a granular material during flow in contact with a selected material. A sample of $50 \mathrm{~g}$ of powder is poured manually (the feeding can be performed automatically too) in a V-tube and flows to a Faraday cup. The V-tube is an assembly of two tubes of length $\mathrm{L}=350 \mathrm{~mm}$ and internal diameter $\mathrm{D}=47 \mathrm{~mm}$ to form a V-shape with an angle of $90^{\circ}$. For the present study, the two tubes forming the $\mathrm{V}$-shape are made of the same material and are attached to each other. Stainless steel $316 \mathrm{~L}$ (SS) and acrylonitrile butadiene styrene (ABS) have been selected. The Faraday cup is connected to a customized electrometer able to measure electrostatic charges. At the end of the flow, the total value of the electric charge Q present in the powder is measured and the charge density $\mathrm{q}=\mathrm{Q} / \mathrm{m}$, where $\mathrm{m}$ is the sample mass, is computed. With each sample, we checked that the electrostatic charge present in the powder

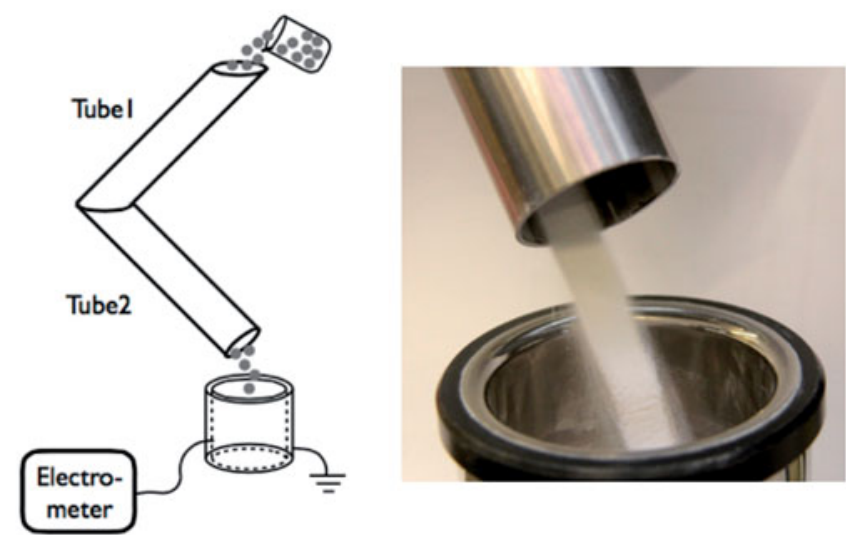

Figure 3. (left) Sketch of the powder tribocharger (GranuCharge from GranuTools) used to measure the electrostatic charge created inside a powder after flow in contact with a selected material. (right) Picture of the powder flowing from the Tube 2 into the Faraday Cup measuring the electrostatic charge.

before the flow in the $\mathrm{V}$-tube is negligible compared to the charge after a flow in the $\mathrm{V}$-tube. The $\mathrm{V}$-tube geometry has been selected to combine the different mechanisms leading to tribo-electrification: (i) friction between the grains, (ii) friction between the grains and the wall and (iii) impact of the grains on the wall at the connection between the two tubes. Therefore, the V-tube charges the powder more in comparison to a single tube geometry, Peltonen, Murtomaa, 
and Salonen (2018). This device is presently commercialized by GranuTools under the name GranuCharge.

At the exception of the results presented in Figure 4, all the measurements presented in the present article have been performed at a relative air humidity of $35 \%+/-5 \%$ and at a temperature of $24^{\circ} \mathrm{C}+/-3{ }^{\circ} \mathrm{C}$. These variations of both relative air humidity and temperature lead to a significant variation of the absolute air humidity. In normal condition of pressure, the absolute humidity range from 5 to 13 gram of water per $\mathrm{m}^{3}$ of air. Therefore, we expect fluctuations in the results due to this humidity variation during the measurement campaign. Performing the measurements in a more controlled environment will reduce the error bars. However, it will not change the general conclusions of the present article.

Before each measurement, the tubes were washed with analytical grade ethanol and dried with a flow of dry air. Afterward, the tubes were used after a waiting time of at least 12 hours in ambient conditions to reach an equilibrium and to dissipate residual electrostatic charges in the case of ABS.

\section{Results and discussion}

\subsection{APIs and excipients tribo-electrification}

Figure 5 shows the charge densities $q$ obtained with the complete set of excipients and APIs in contact with both

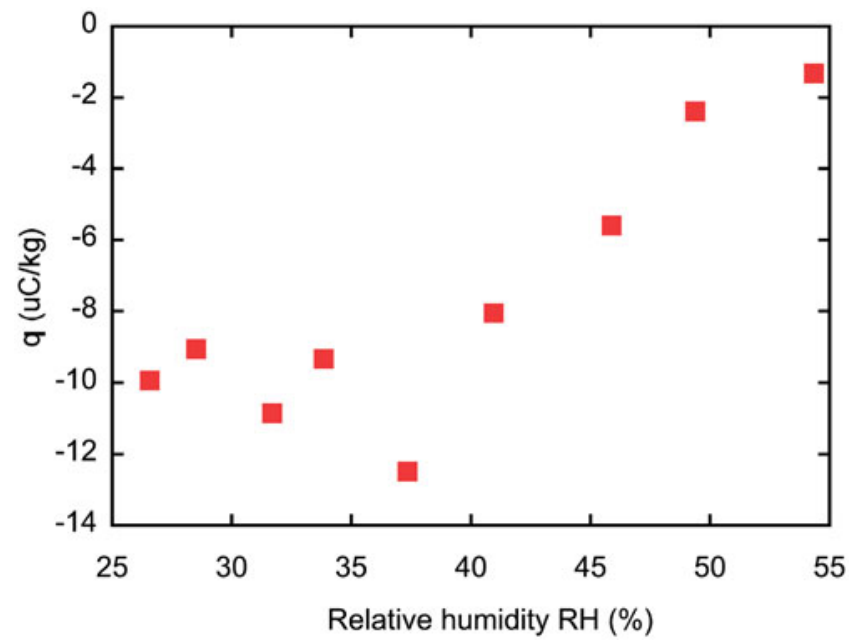

Figure 4. Evolution of the charge density q for a lactose powder (Inhalac 230) in contact with SS as a function of relative air humidity. stainless steel (left) and ABS (right). The powders are numbered (see Table 1) and are within their category sorted (from the smallest to the highest charge, taking the sign into account) as a function of their electrification against stainless steel. Table 2 gives the main granulometric parameters with the same sorting. The different excipients have almost the same triboelectric behavior and a low chargeability. With excipients, we do not observe any correlation between the chargeability and the grain size (see Table 2). Moreover, the different excipient families are not evidenced by the chargeability. It has to be noted that chemically speaking, all excipients used in this study are saccharides or polysaccharides and hence contain very similar chemical groups, mainly $\mathrm{C}-\mathrm{OH}$ moieties.

Concerning the APIs, some of them charge a lot while other APIs charge less. Moreover, the sign of the charges varies from one API to the other. This variety of triboelectric behavior for APIs in contact with stainless steel has been already evidenced by Supuk et al. 2012. It is interesting to notice that the APIs with similar chemical structures present similar triboelectric behavior. Indeed, when in contact with the SS, the three APIs of the "zine" family (powders number 21-23) present the highest chargeability properties whereas the three APIs from the "racetam" family (powders number 16-18) have a low-charging propensity. In addition, for APIs, a higher chargeability is observed with smaller grains. Figure 6 shows the absolute value of the charge density $|\mathrm{q}|$ obtained with APIs against stainless steel as a function of the average grain size $\mathrm{d}(\mathrm{v}, 0.5)$. A global decrease of the chargeability is observed when the average grain size increases. Therefore, the triboelectric effect seems to be linked to both the global chemical structure of the molecule and the grain size. The effect of grain size is probably linked to the higher surface area and number of contacts available for charging when particle size decreases.

The excipients charge negatively against SS and positively against ABS. Figure 5 does not show any clear correlation between the chargeability against SS and against ABS. Globally, the powders are acquiring more charges when flowing against SS. SS is an electrical conductor while ABS is an insulating material. During the measurement, the SS tubes are grounded like in the majority of the processing devices in the industry. On the contrary, the charges created at the internal surface of the ABS tube are only slowly dissipated. The results might confirm recent studies showing
On stainless steel

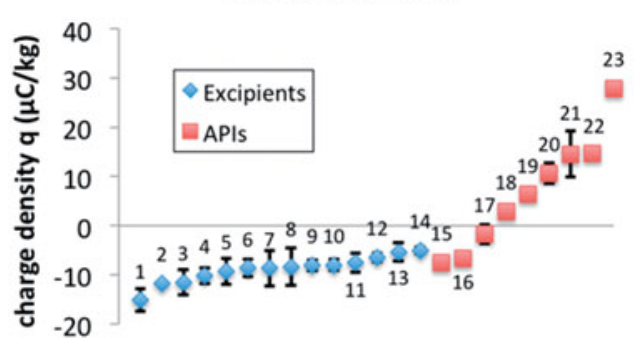

On ABS

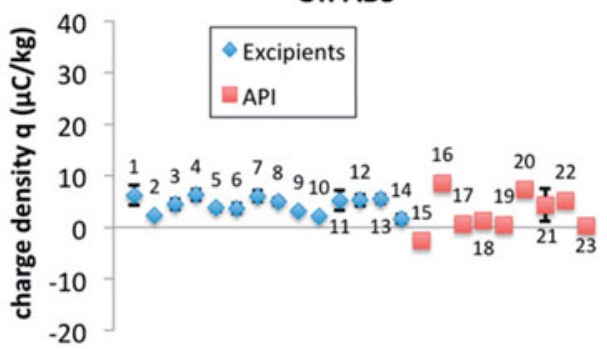

Figure 5. Charge densities $\mathrm{q}=\mathrm{Q} / \mathrm{m}$ expressed in $\mu \mathrm{C} / \mathrm{kg}$ and obtained with the complete set of excipients and APIs in contact with both stainless steel (left) and ABS (right). The powders are numbered (see Table 1) and sorted (from the smallest to the highest charge, taking the sign into account) as a function of their electrification against stainless steel. The error bars correspond to standard deviations computed on a minimum of three measurements. 
that common practice like grounding to avoid powder electrostatic charging is less effective than previously thought, Karner and Urbanetz (2012).

In Figure 5, error bars corresponding to the standard deviation with a minimum of three measurements are presented for the whole set of excipients at the exception of excipient number 2 (InhaLac 230) that was measured only once. Concerning the APIs, error bars are presented for the three powders (Levetiracetam, Lacosamide, and Cetirizine) considered in mixtures (see hereafter). The variability of these results is mainly related to the variability of the absolute air humidity. Indeed, the measurements were performed during a period of six months in a partially conditioned lab.

\subsection{Influence of air humidity}

Before considering the blends, we would like to emphasize the influence of relative air humidity (RH) on the tribocharging mechanism. Figure 4 shows the charge density $\mathrm{q}$ obtained with a lactose powder for different $\mathrm{RH}$ values (at a constant temperature of $22^{\circ} \mathrm{C}+/-1^{\circ} \mathrm{C}$ ) against SS.

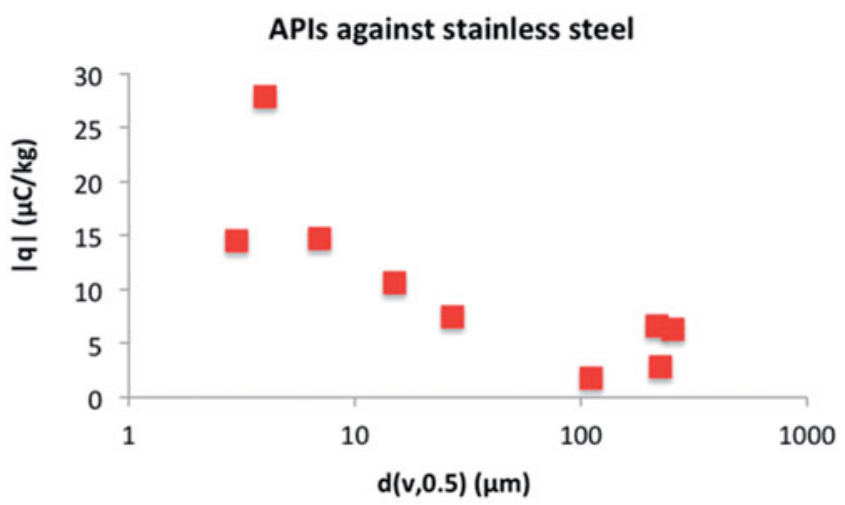

Figure 6. Absolute value of the charge density $|q|$ obtained with APIs against stainless steel as a function of the average grain size $d(v, 0.5)$.
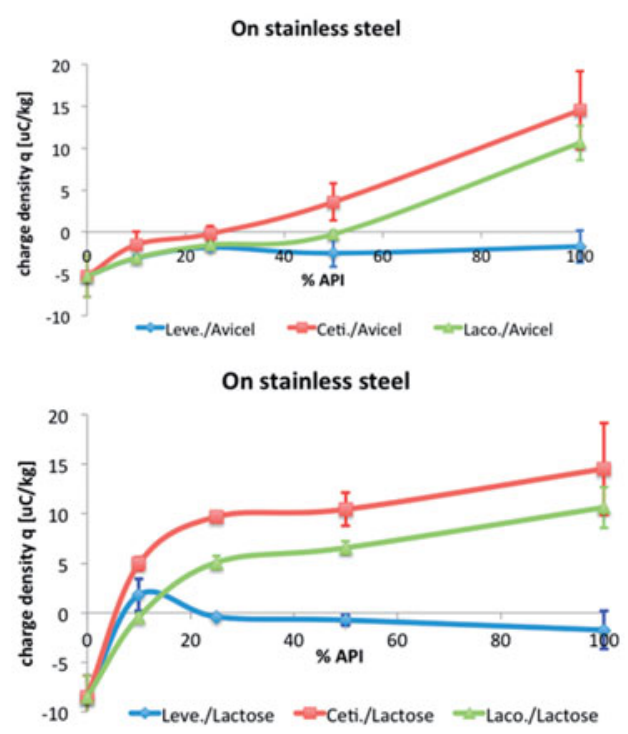

The different $\mathrm{RH}$ values have been obtained by switching off the humidity regulator of the laboratory. Therefore, we took advantage of the Belgian meteorological fluctuations. The absolute value of the charge density remains relatively constant from 25 up to $40 \% \mathrm{RH}$ but decreases drastically increasing $\mathrm{RH}$ from 40 to $50 \%$. This observation is in agreement with the results obtained very recently by Schella, Herminghaus, and Schroter (2017) with a different tribocharger, i.e. a shaken cell. The charge decrease at high RH is certainly a consequence of the surface resistivity decrease induced by the presence of condensed water at the surface of the grains.

\subsection{Tribo-electrification of API/excipient blends}

Two excipients (Avicel PH102 and Lactose Tablettose 80) and three APIs (Levetiracetam, Cetirizine, and Lacosamide) have been selected to investigate the tribo-electric behavior of blends. For each excipient/API combination, three proportions of API content have been considered: 10, 25, and $50 \%$. The blends were prepared in a rotating V-shape mixer (Filtra, FTLMV-02) during 15 minutes. The obtained charge density in contact with both stainless steel and ABS are presented in Figure 7.

The charge density of the mixtures is found to depend strongly on the material in contact (SS or ABS), showing the importance of the possibility to use the same material in the lab as in the process line. The charge density of the mixtures is globally lower than the charge density of the API alone. However, contrary to the results presented in previous studies, Naik, Mukherjee, and Chaudhuri (2016), the charge density of the mixtures is not necessarily drastically lower than the charge density of the components. For some blends, we observe a monotonic evolution of the charge density as a function of the API content. In contact with SS, the charge density of the mixtures Ceti./Avicel, Laco./Avicel, Ceti./Lactose, and Laco./Lactose increases from the negative
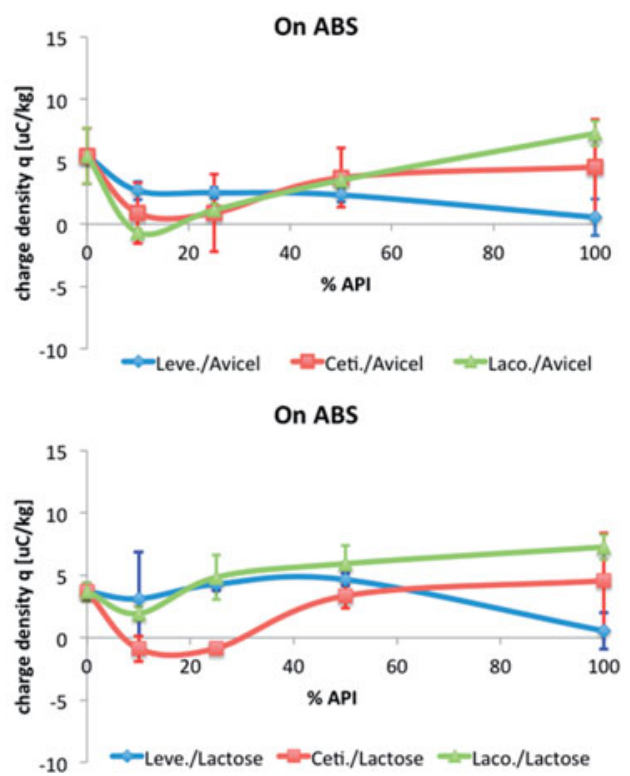

Figure 7. Charge density q inside excipient/API blends after flow in contact with SS (left) and ABS (right). The combinations between Avicel PH102 and Tablettose 80 excipients and Levetiracetam, Lacosamide and Cetirizine APIs are considered. 
value corresponding to the excipient alone to the positive value corresponding to the API alone. For other blends, the charge density evolution as a function of the API content is non-monotonic. In contact with SS, the curve corresponding to the mixture Leve./Lactose starts with a negative charge with pure Lactose, becomes positive for low API content and finishes negative for the API alone.

As shown by Table 2 and Figure 2, the excipients are free-flowing powders made of relatively large grains while APIs are highly cohesive powders made of smaller grains (at the exception of Levetiracetam). Therefore, the structure of the mixtures is complex with the smaller API grains sticking on the larger excipient grains. In particular, this effect is expected with blends containing Cetirizine and Lacosamide. Moreover, for higher API content, the small API grains are expected to form agglomerates. In addition, we measure the total charge in the sample while we expect to have a charge distribution in the pile. This distribution of charge could be particularly complex in the case of a mixture of positive and negative grains. For these reasons, the net chargeability of a powder blend is extremely difficult to predict with a bottom-up method and a measurement is necessary.

The coating of the device by a constituent could influence the final blend charge, Elajnaf, Carter, and Rowley (2006), Naik, Mukherjee, and Chaudhuri (2016). With our measurement method, the effect of this coating is very limited because we measure the powder charge after the first flow in a clean V-tube. The effect of the coating could be investigated by making successive powder flows in the V-tube, but this is outside the scope of the present article.

\section{Conclusion}

The tribo-electrification of excipients and APIs have been analyzed while flowing in contact with stainless steel and ABS. The obtained charge density is found to depend on the material in contact. Moreover, the charge density is globally more important with APIs. APIs with similar chemical structures present similar triboelectric behavior. In addition, for APIs, a link between grain size and chargeability is observed, small grains charge more. Therefore, their tribocharging behaviors are influenced by both chemical structure and grain size.

The tribo-electrification is found to depend drastically on hygrometric conditions of the air. For relative air humidity values higher than 50\%, the tribo-electrification is reduced in case of lactose monohydrate. Controlling more precisely the hygrometric conditions during the measurement constitute a room of improvement for the present study.

For some excipient/API blends, we observe a monotonic evolution of the charge density as a function of composition. However, for some blends, the charge density evolution as a function of the API content is non-monotonic and unexpected sign changes are observed.

Tribo-electric charging of powders is a complex mechanism influenced by a wide range of parameters: surface nature (material, roughness, and surface cleanness), grain properties (chemical nature, grain size distribution, grain shape and roughness, grain crystallinity) and environmental conditions (RH). A complete explanation of the results needs a deeper understanding of the triboelectric process in powder and fundamental studies are still necessary. Finally, the present work evidences the necessity to perform a measurement because the prediction of the triboelectric behavior of a blend is highly complex, in fact practically impossible.

\section{Acknowledgments}

We thank Bruno Chaumont, Coralie Renders, Gregory Descamps, Sandrine Rome, and Anne Bouquelle from UCB Pharma for their technical support.

\section{Funding}

This work was supported by the Walloon Region of Belgium (BEWARE GRANELEC Ref:1410265).

\section{References}

Baltes, E., J. De Lannoy, and L. Rodriguez. 1985. 2-[4-(Diphenylmethyl)1-piperazinyl]-acetic acids and their amides. US patent 4,525,358, issued June 25, assigned to UCB Pharmaceuticals, Inc.

Boschini, F., V. Delaval, K. Traina, N. Vandewalle, and G. Lumay. 2015. Linking flowability and granulometry of lactose powders. International Journal of Pharmaceutics 494 (1):312-20. doi:10.1016/ j.ijpharm.2015.08.030.

Bunten, S., and S. Happe. 2006. Rotigotine transdermal system: A short review. Neuropsychiatr Dis Treat 2 (4):421-6.

Elajnaf, A., P. Carter, and G. Rowley. 2006. Electrostatic characterisation of inhaled powders: Effect of contact surface and relative humidity. European Journal of Pharmaceutical Sciences: Official Journal of the European Federation for Pharmaceutical Sciences 29 (5):375-84. doi:10.1016/j.ejps.2006.07.006.

Engers, D. A., M. N. Fricke, A. W. Newman, and K. R. Morris. 2007. Triboelectric charging and dielectric properties of pharmaceutically relevant mixtures. Journal of Electrostatics 65 (9):571-81. doi: 10.1016/j.elstat.2006.12.002.

Genton, P., and B. Van Vleymen. 2000. Piracetam and levetiracetam: Close structural similarities but different pharmacological and clinical profiles. Epileptic Disorders: International Epilepsy Journal with Videotape 2 (2):99-105.

Ghori, M. U., E. Šupuk, and B. R. Conway. 2015. Tribo-electrification and powder adhesion studies in the development of polymeric hydrophilic drug matrices. Materials (Basel, Switzerland) 8 (4): 1482-98. doi:10.3390/ma8041482.

Glor, M. 2003. Ignition hazard due to static electricity in particulate processes. Powder Technology 135-136:223-33. doi: 10.1016/j.powtec.2003.08.017.

Guaiana, G., C. Barbui, and A. Cipriani. 2010. Hydroxyzine for generalised anxiety disorder. Cochrane Database Syst Rev (12):CD006815. doi:10.1002/14651858.CD006815.pub2.

Hoy, S. M. 2013. Lacosamide: A review of its use as adjunctive therapy in the management of partial-onset seizures. CNS Drugs 27 (12): 1125-42. doi:10.1007/s40263-013-0123-5.

Ireland, P. M. 2010. Triboelectrification of particulate flows on surfaces: Part I - Experiments. Powder Technology 198:189-98. doi:10.1016/ j.powtec.2009.11.017.

Ireland, P. M., and K. Nicholson. 2011. Analysis and comparison of particle tribochargers. Minerals Engineering 24 (8):914. doi:10.1016/ j.mineng.2011.04.006.

Karner, S., and N. A. Urbanetz. 2012. Arising of electrostatic charge in the mixing process and its influencing factors. Powder Technology 226:261-8. doi:10.1016/j.powtec.2012.04.062. 
Lachiver, E. D., N. Abatzoglou, L. Cartilier, and J.-S. Simard. 2006. Insights into the role of electrostatic forces on the behavior of dry pharmaceutical particulate systems. Pharmaceutical Research 23 (5): 997-1007. doi:10.1007/s11095-006-9934-0.

Lacks, D. J. 2010. Particle clouds: Frictile attraction. Nature Physics 6 (5):324-5. doi:10.1038/nphys1660.

Lumay, G., F. Boschini, K. Traina, S. Bontempi, J.-C. Remy, R. Cloots, and N. Vandewalle. 2012. Measuring the flowing properties of powders and grains. Powder Technology 224:19-27. doi:10.1016/j.powtec.2012.02.015.

Matagne, A., D. G. Margineanu, B. Kenda, P. Michel, and H. Klitgaard. 2008. Anti-convulsive and anti-epileptic properties of brivaracetam (ucb 34714), a high-affinity ligand for the synaptic vesicle protein, SV2A. British Journal of Pharmacology 154 (8):1662-71. doi:10.1038/ bjp.2008.198.

Matsusaka, S., H. Maruyama, T. Matsuyama, and M. Ghadiri. 2010. Triboelectric charging of powders: a review. Chemical Engineering Science 65 (22):5781-807. doi:10.1016/j.ces.2010.07.005.

Mehrani, P., M. Murtomaa, and D. J. Lacks. 2017. An overview of advances in understanding electrostatic charge buildup in gas-solid fluidized beds. Journal of Electrostatics 87:64-78. doi:10.1016/ j.elstat.2017.03.005.

Mersch, E., G. Lumay, F. Boschini, and N. Vandewalle. 2010. Effect of an electric field on an intermittent granular flow. Physical Review. E, Statistical, Nonlinear, and Soft Matter Physics 81 (4 Pt 1):041309. doi:10.1103/PhysRevE.81.041309.

Murtomaa, M., and E. Laine. 2000. Electrostatic measurements on lactose-glucose mixtures. Journal of Electrostatics 48 (2):155-62. doi: 10.1016/S0304-3886(99)00063-7.

Muzzio, F. J., T. Shinbrot, and B. J. Glasser. 2002. Powder technology in the pharmaceutical industry: The need to catch up fast. Powder Technology 124 (1-2):1-7. doi:10.1016/S0032-5910(01)00482-X.

Naik, S., R. Mukherjee, and B. Chaudhuri. 2016. Triboelectrification: A review of experimental and mechanistic modelling approaches with a special focus on pharmaceutical powders. International Journal of Pharmaceutics 510 (1):375-85. doi:10.1016/j.ijpharm.2016.06.031.

Ohsawa, A. 2011. Statistical analysis of fires and explosions attributed to static electricity over the last 50 years in Japanese industry. Journal of Physics: Conference Series 301:012033.
Peltonen, J., M. Murtomaa, and J. Salonen. 2018. Measuring electrostatic charging of powders on-line during surface adhesion. Journal of Electrostatics 93:53-7. doi:10.1016/j.elstat.2018.03.007.

Rogawski, M. A. 2008. Brivaracetam: A rational drug discovery success story. British Journal of Pharmacology 154 (8):1555-7. doi:10.1038/ bjp.2008.221.

Sarkar, S., R. Mukherjee, and B. Chaudhuri. 2017. On the role of forces governing particulate interactions in pharmaceutical systems: a review. International Journal of Pharmaceutics 526 (1-2):516-37. doi:10.1016/j.ijpharm.2017.05.003.

Schella, A., S. Herminghaus, and M. Schroter. 2017. Influence of humidity on tribo-electric charging and segregation in shaken granular media. Soft Matter 13 (2):394-401. doi:10.1039/ c6sm02041k.

Shaw, I. C., and M. I. Graham. 1987. Mesna-a short review. Cancer Treatment Reviews 14 (2):67-86. doi:10.1016/0305-7372(87)90041-7.

Šupuk, E., A. Zarrebini, J. P. Reddy, H. Hughes, M. M. Leane, M. J. Tobyn, P. Timmins, and M. Ghadiri. 2012. Tribo-electrification of active pharmaceutical ingredients and excipients. Powder Technology 217:427-34. doi:10.1016/j.powtec.2011.10.059.

van de Walle, J., P. Lauwers, and H. Adriaensen. 1976. Mistabron in the intensive care unit. Acta Anaesthesiologica Belgica 27:319-21.

Waitukaitis, S. R., V. Lee, J. M. Pierson, S. L. Forman, and H. M. Jaeger. 2014. Size-dependent same-material tribocharging in insulating grains. Physical Review Letter 112:218001. doi:10.1103/ PhysRevLett.112.218001.

Wong, J., H. K. Chan, and P. C. L. Kwok. 2013. Electrostatics in pharmaceutical aerosols for inhalation. Therapeutic Delivery 4 (8): 981-1002. doi:10.4155/tde.13.70.

Wong, J., P. C. L. Kwok, and H.-K. Chan. 2015. Electrostatics in pharmaceutical solids. Chemical Engineering Science 125:225-37. doi: 10.1016/j.ces.2014.05.037.

Zafar, U., F. Alfano, and M. Ghadiri. 2018. Evaluation of a new dispersion technique for assessing triboelectric charging of powders International Journal of Pharmaceutics 543 (1-2):151-9. doi:10.1016/ j.ijpharm.2018.03.049. 